\title{
Penile traction therapy in Peyronie's disease
}

\section{Jason M Greenfield}

\author{
Address: Department of Urology, Columbia University Medical Center, The Herbert Irving Pavilion, 161 Fort Washington Avenue, 11th Floor, \\ New York, NY 10032, USA \\ Email: jg2793@columbia.edu
}

FI000 Medicine Reports 2009, I:37 (doi:I0.34I0/MI-37)

The electronic version of this article is the complete one and can be found at: http://FI000.com/Reports/Medicine/content/I/37

\begin{abstract}
Penile traction therapy has traditionally been implemented to increase penile length but has recently been investigated for reducing the curvature associated with Peyronie's disease. The results of a few initial investigations have been conflicting, and further research is needed to confirm the true benefit of such therapy and its potential role in treating Peyronie's disease as both a monotherapy and in combination with other therapeutic options.
\end{abstract}

\section{Introduction and context}

Peyronie's disease (PD) is a connective tissue disorder involving the tunica albuginea layer of the penis. The fibrotic process that occurs may result in the formation of plaque, one of the classic findings on physical examination of the patient. Men afflicted by this disorder may present with pain, deformity (such as penile curvature or penile shortening) and/or erectile dysfunction (ED). While surgical therapy has been the standard definitive treatment for penile curvature associated with $\mathrm{PD}$, less invasive options have been sought. Aside from the common risks associated with surgical procedures, corrective surgery for the penile curvature associated with PD can result in further penile shortening and worsening penile rigidity with erection [1-4]. Medical options are attractive due to their potential to avoid invasive procedures of the penis, to affect all aspects of the disease, and to intervene before the disease has stabilized and is no longer reversible. However, no single medical treatment has been definitively proven to offer a significant benefit in a large placebo-controlled trial $[5,6]$. In addition, no medical or surgical therapy offers the benefit of increased penile length to men with PD, a common sequela of the disease and one that can cause significant emotional distress.

While the search for an effective non-surgical therapy for PD has continued, interest in the use of external mechanical force has arisen. The use of a vacuum erection device (VED) has been recommended as an option for treatment of the shortening, curvature, and ED associated with PD. It has also been investigated as an adjunctive therapy to be used in combination with surgical treatment of PD [7]. To date, no large multicenter controlled trial has been performed to validate the use of a VED in these situations.

While the use of traction therapy in PD may be a relatively new concept, it has a long history of use in other areas of medicine. In 1969, Ilizarov and Soibeman [8] described 'distraction osteogenesis' as a technique to stimulate bone remodeling. Subsequently, the use of traction and tissue expansion therapy has spread to other areas, including orthodontics and plastic/reconstructive surgery [9]. From these initial experiences, the concept of using traction therapy to lengthen the penis was felt to be a logical consideration. In 2001, Scroppo and colleagues [10] reported the results of a small study that investigated the use of penile traction to treat PD curvature. Eight men with PD and no complaints of ED were instructed to use a penile traction device for at least 4 hours per day for a total treatment period of 3-6 months. The authors reported a statistically significant decrease in mean erect curvature of $14^{\circ}$ (from 34 to $20^{\circ}$ ). However, this study involved a very small cohort of patients with no control group. 
Several years later, Moncada-Iribarren and colleagues [11] reported on the use of a traction device to treat penile shortening that had occurred after PD surgery. A total of 40 men participated in this study, with 12 undergoing a grafting procedure while the remaining 28 underwent plication only. One-half of the patients were treated with penile traction and the other half served as the control group. The penile extender was first instituted once the surgical incision had healed (approximately 2-3 weeks) for 8-12 hours daily for a total treatment period of at least 4 months. For both groups, penile shortening after surgery ranged from $0.5-4.0 \mathrm{~cm}$. Those patients in the treatment group experienced a length increase ranging from $1-3 \mathrm{~cm}$ and this increase was proportional to the number of hours per month that the patient was wearing the extender. The authors also studied the potential impact of the device on the quality of life of their study groups and identified significant differences in several parameters for the patients using the device versus those in the control group.

\section{Recent advances}

Based on these initial positive studies, further investigation into the use of penile traction as a non-surgical alternative in the treatment of PD deformity was encouraged. In 2008, Levine and colleagues [12] reported the results of a study of 11 men with PD who underwent treatment with a penile traction device. All participants were evaluated with pre-treatment measurement of stretched penile length from pubis to corona, and a dynamic color duplex ultrasound was performed with measurement of curvature and girth at maximal erection. Of the 11 men who entered the study, one subject dropped out due to inability to comply with the treatment protocol. Patients were instructed to wear the device for a minimum of 2 hours per day but were encouraged to increase the duration of use to a maximum of 8 hours per day. Treatment was continued for a total of 6 months. Every 2 weeks, the extender rods were lengthened by $0.5 \mathrm{~cm}$, and repeat length measurements were recorded every month during an office visit.

Of the 10 men who completed the study, all reported subjective improvements in length and curvature of $0.5-2.5 \mathrm{~cm}$ and $10-40^{\circ}$, respectively. Objectively, mean penile stretched length increased from $0.5-2.0 \mathrm{~cm}$ and curvature had been reduced by $10-45^{\circ}$ (mean of $22^{\circ}$ ). There were no patient-described changes regarding penile sensation, worsening erectile function, or skin injury. Overall, patients reported high satisfaction rates and improvements in International Index of Erectile Function (IIEF) scores.
This year, Gontero and colleagues [13] published the results of a study of 19 men who underwent treatment with a penile traction device for PD-associated penile curvature. Of the men participating, all had pretreatment curvature of less than $50^{\circ}$, disease lasting a minimum of 12 months, and no penile pain when flaccid. In contrast to the Levine study, measurements of curvature were determined by photography taken by the investigators after a pharmacologically induced erection in the office or, for patients who refused, by autophotography at home. Patients were required to wear the device for a minimum of 5 hours daily, up to a maximum of 9 hours. Patients were evaluated at months 1,3 , and 6 . After finishing treatment at month 6 , they were evaluated again at 12 months (washout period of 6 months). A total of four patients were dropped from the final analysis due to lack of compliance with the protocol or were lost to follow-up. For the remaining 15 patients available for analysis, median daily use of the device was 5.5 hours. Penile curvature decreased from a mean of $31^{\circ}$ to $27^{\circ}$. Although this was not statistically significant, there was a significant improvement in mean flaccid and stretched penile length measurements of 1.3 and $0.83 \mathrm{~cm}$, respectively. No further changes in curvature or length were noted after the washout period. There was only a marginal improvement in IIEF score, which was also not statistically significant.

There are several potential explanations for the large discrepancies in the findings between the Levine and Gontero studies. The selection criteria for each study were quite different. For example, the Gontero study required all patients to have the stable form of the disease, which possibly meant that this patient group had disease symptoms that were less amenable to therapy. The Gontero study also limited their study group to patients with curvature of less than $50^{\circ}$, which may have reduced the potential impact of traction therapy. In addition, it is difficult to compare changes in IIEF scores as the treatment group from both studies differed in their baseline scores. Treatment protocol and methods of evaluation were not kept constant across both studies.

\section{Implications for clinical practice}

While the value of traction forces in treating various medical conditions has been established, its role in the treatment of the deformities associated with PD has yet to be defined. Initial studies have indicated a role in promoting recovery of penile length lost as a result of the disease. More recent investigations have validated this, although a large controlled trial has yet to be performed. Of the few studies that have been done, contrasting evidence exists as to how beneficial a traction device may 
be for improving penile curvature. While some mild to moderate improvement may be anticipated, a lack of uniformity in the evaluation of patients across these studies causes the results to be difficult to interpret. Until uniform methods of evaluating PD patients are agreed upon across these studies, a definitive answer will be difficult to reach [14].

As the search for effective non-surgical therapies for PD continues, some have proposed a role for 'combination therapy', with traction therapy being a potential component. In fact, Abern and Levine [15] reported on such a protocol in a study of 71 men. In this non-controlled study, a greater improvement in penile curvature was seen in those patients who elected to undergo verapamil injection along with penile traction therapy as opposed to those who underwent injection therapy alone. Again, no definitive recommendations for changes in clinical practice can be made from these findings, but similar studies are forthcoming. Until these investigations involve larger cohorts in a controlled study, it will continue to prove difficult for definitive recommendations to be made.

The investigation of the use of traction therapy in treating men with PD is still in its infancy. Although no large multi-center controlled trials have been published to date, the initial reports are promising. Whether traction therapy will prove beneficial as a monotherapy or in combination with other therapies remains to be seen. At this point, penile traction appears to be a safe and potentially effective option for men wishing to minimize loss of length after corrective surgery for PD. It may also be a reasonable alternative for men who are not candidates for surgery or refuse surgery, and who are wishing to seek modest improvements in recovery of length and penile curvature.

\section{Abbreviations}

$\mathrm{ED}$, erectile dysfunction; IIEF, International Index of Erectile Function; PD, Peyronie's disease; VED, vacuum erection device.

\section{Competing interests}

The author declares that he has no competing interests.

\section{References}

I. Van Der Horst C, Martinez Portillo FJ, Seif C, Alken P, Juenemann KP. Treatment of penile curvature with Essed-Shroeder tunical plication: aspects of quality of life from the patient's perspective. BJU Int 2004, 93:105-08.

2. Syed AH, Abbasi Z, Hargreave TB: Nesbit procedure for disabling Peyronie's curvature: a median follow-up of 84 months. Urology 2003, 61:999-1003.

3. Greenfield JM, Lucas S, Levine LA: Factors affecting the loss of length associated with tunica albuginea plication for correction of penile curvature. J Urol 2006, I 75:238-4I.

4. Breyer BN, Brant WO, Garcia MM, Bella AJ, Lue TF: Complications of porcine small intestine submucosa graft for Peyronie's disease. J Urol 2007, 177:589-91.

5. Greenfield JM: Peyronie's disease: options in pharmacologic management. Exp Review Clin Pharm 2008, I:299-306.

6. Gholami SS, Gonzalez-Cadavid NF, Lin CS, Rajfer J, Lue TF: Peyronie's disease: a review. J Urol 2003, I69:।234-4I.

7. Lue TF, El-Sakka $\mathrm{Al}$ : Lengthening shortened penis caused by Peyronie's disease using circular venous grafting and daily stretching with a vacuum erection device. J Urol 1999, | 6 |: | | $4 \mid-4$.

8. Ilizarov GA, Soibeman LM: Clinical and experimental data on bloodless lengthening of lower extremities. Eksp Khir Anesteziol 1969, 14:2D-8D

9. Messina A, Messina J: The continuous elongation treatment by the TEC device for severe Dupuytren's contracture of the fingers. Plast Reconstr Surg 1993, 92:84-90.

10. Scroppo FI, Mancini M, Maggi M, Colpi GM: Can an external penis stretcher reduce Peyronie's penile curvature? Int J Impot Res 2001, I3 (Suppl 4).

II. Moncada-Iribarren I, Jara J, Martinez-Salamanca Jl, Cabello R, Hernandez C: Managing penile shortening after Peyronie's disease surgery. J Urol 2007, I77 (Suppl 4):252.

12. Levine LA, Newell M, Taylor FL: Penile traction therapy for treatment of Peyronie's disease: A single-center pilot study. J Sex Med 2008, 5:1468-73.

13. Gontero P, Di Marco M, Giubilei G, Bartoletti R, Pappagallo G, Tizzani $A$, Mondani $N$ : Use of penile extender device in the treatment of penile curvature as a result of Peyronie's disease. Results of a phase II prospective study. J Sex Med 2009, 6:558-66

14. Levine LA, Greenfield JM: Establishing a standardized evaluation of the man with Peyronie's disease. Int J Impot Res 2003, I5 (Suppl 5): SI03-12.

15. Abern MR, Levine LA: Intralesional verapamil injections with and without penile traction and oral therapies for management of Peyronie's disease. J Urol 2008, 179 (Suppl 4):408. 